Mediterránea Ser. Biol. (1992/93), n. ${ }^{\circ}$ 14. Pág. 79-102

\title{
LA PROBLEMÁtICA DE LOS ROEDORES COMENSALES EN LA VEGA BAJA DEL RÍO SEGURA (ALICANTE) ${ }^{1}$
}

\author{
por \\ F.V. FAUS ${ }^{2}$
}

\section{RESUMEN}

Este trabajo aborda la problemática originada por los roedores comensales en la Vega Baja del río Segura (Alicante, España). La metodología básica utilizada ha consistido en el muestreo del-medio físico, tanto urbano como rural, recopilándose las observaciones directas e indirectas de los animales que han permitido elaborar los resultados más importantes. Así, se confirma la presencia de especies típicamente antropófilas como Rattus norvegicus Berk., Rattus rattus L. y Mus musculus L., y otras de tendencias más silvestres como Arvicola sapidus Mill. y Mus spretus Lat.. Se analiza su distribución geográfica, la ocupación del medio físico urbano y rural, el nivel de infestación en los emplazamientos inspeccionados de cada municipio, los dańos causados a nivel cualitativo y la información recogida en una encuesta realizada sobre el tema. El roedor predominante es $R$. norvegicus, aunque la extensa red de canales de riego y el carácter eminentemente agrícola de la comarca favorecen la dispersión de todas las especies. Las alternativas de solución pasan por la adopción de medidas como la realización de programas de control de roedores mediante técnicas químicas y biológicas, al igual que el saneamiento general de los cursos de agua, sobre todo del río Segura, la desaparición de los vertederos incontrolados y la colaboración de los lugareños.

PALABRAS ClAVE: Roedores comensales, problemas, Vega Baja del río Segura, Alicante, España.

\section{SUMMARY}

This work tackles the problem caused by the commensal rodents on the Lower Meadow of the Segura River (Alicante, Spain). The basic methodology used consists of the survey of the physical environment, both urban an rural, by means of the compilation of direct an indirect observations of those animals that have enabled to achieve the most important results. Thus, we can confirm the diffusion of species that are typically anthropophilous such as Rattus

${ }^{i} \mathrm{La}$ información contenida en este artículo ha formado parte de dos comunicaciones presentadas en la VII Reunión Bienal de la Real Sociedad Española de Historia Natural (Barcelona, 16-20 Septiembre 1985).

${ }^{2}$ Unidad de Ecología, Departamento de Microbiología, Facultad de C.C. Biológicas, Universitat de València, $\mathrm{C} /$. Dr. Moliner, 50, 46100 Burjasot, Valencia. 
norvegicus Berk., Rattus rattus y L. and Mus musculus L., and the presence of other species that have more wild tendencies like Arvicola sapidus Mill. and Mus spretus Lat.. Their geographical distribution, the occupation of the urban and rural environments, the infestation level in the inspected premises within each municipality, the damage caused and the information collected through a public opinion poll taken on the subject, are studied. The predominant rodent is $R$. norvegicus, althoyugh the extensive network of irrigation channels and the eminently agricultural characteristics of the territory favour the dispersion of the different species. The alternatives for the solution of the problem require the consideration of measures such as the monitoring of rodent control programmes with the help of biological and chemical techniques, as well as the general improvement of the watercourses, especially those of the Segura River, the eradication of uncontrolled urban dumping grounds and the collaboration of the inhabitants from all municipalities.

KEY WORDS: Commensal rodents, problem, Lower Meadow of Segura River, Alicante, Spain.

\section{INTRODUCCIÓN}

La capacidad que tienen algunos animales para vivir a expensas del hombre y competir con él por los alimentos y el medio físico se llama comensalismo. Los roedores comensales merecen especial atención porque a nivel mundial causan daños en las cosechas y transmiten enfermedades al ganado doméstico y a las personas (BROOKS, 1973; WORLD HEALTH ORGANIZATION, 1974; ARATA, 1977; BROOKS y ROWE, 1979; GRATZ, 1984). Entre las especies más importantes destacan la rata parda (Rattus norvegicus Berkenhout, 1769), la rata negra (Rattus rattus Linnaeus, 1758) y el ratón doméstico (Mus musculus Linnaeus, 1758).

Por su facilidad de adaptación a cualquier condición de vida y sus tendencias antropófilas, $R$. norvegicus ocupa en Europa gran variedad de biotopos tanto en zonas urbanas como rurales (BROOKS y ROWE, 1979). En la Península Ibérica, esta especie aumenta su carácter antropófilo desde la costa mediterránea hasta los Pirineos donde se limita a invadir las casas (GOSÀLBEZ, 1979). Mientras, $R$. rattus es menos comensal en la región mediterránea que en Centroeuropa (JIRSIK, 1955; TELLE, 1966), caracterizándose por sus hábitos arborícolas (KAHMANN y HAEDRICH, 1957; FAUS y VERICAD, 1981; FAUS, 1990). Por último, $M$. musculus es una especie ubiquista que prefiere la cercanía del hombre (SCHWARZ y SCHWARZ, 1943; YALDEN, 1980), aunque también abunda en estado salvaje (VENABLES y LESLIE, 1942; BERRY, 1981; PELIKAN, 1981).

El presente trabajo intenta dar una visión general de la problemática ocasionada por los roedores comensales en el medio urbano y el medio rural de la Vega Baja del río Segura (Alicante). Se refieren las especies determinadas, su distribución geográfica, los emplazamientos ocupados, los daños observados y los resultados de una encuesta elaborada sobre el tema. Además, se aportan sugerencias complementarias de carácter eminentemente práctico para que, junto a futuras campañas de desratización, eviten la aparición de plagas. 


\section{ÁREA DE ESTUDIO}

\section{Situación y extensión}

La Vega Baja del río Segura es la comarca más meridional de la provincia de Alicante y, al mismo tiempo, de la Comunidad Valenciana. El territorio comprende 24 términos municipales de los que ocho poblaciones se sitúan a lo largo del río Segura: Albatera, Algorfa, Almoradí, Benferri, Benejúzar, Benijófar, Bigastro, Callosa de Segura, Catral, Cox, Daya Nueva, Daya Vieja, Dolores, Formentera del Segura, Granja de Rocamora, Guardamar del Segura, Jacarilla, Orihuela, Rafal, Redován, Rojales, San Fulgencio, San Miguel de Salinas y Torrevieja. Estos dos últimos municipios han sido excluidos del trabajo debido a su lejanía del cauce del río. Las coordenadas geográficas extremas están determinadas por las latitudes $37^{\circ} 50^{\prime} 30^{\prime \prime}$ y $38^{\circ} 19^{\prime} 10^{\prime \prime}$, y las longitudes $2^{\circ} 39^{\prime} 00^{\prime \prime}$ y $3^{\circ} 03^{\prime} 05^{\prime \prime}$. La Figura 1 muestra la delimitación municipal, los núcleos de población, las unidades hidrogeográficas y las vías de comunicación más importantes de la misma.

La región abarca una superficie de $973.5 \mathrm{~km} .{ }^{2}$. El número aproximado de habitantes es 151.800 y la densidad de población 156 personas por $\mathrm{km} .{ }^{2}$ (CAJA DE AHORROS DE ALICANTE Y MURCIA, 1980).

\section{Climatología}

El clima típico de la zona es el marítimo del litoral mediterráneo, con veranos calurosos e inviernos templados, alcanzando la temperatura media anual los $18.5^{\circ} \mathrm{C}$ (PANAREDA y NUET, 1979). Los meses más cálidos son julio y agosto, y el más fresco enero. Las épocas de primavera y otoño presentan máximos de precipitación en los meses de abril y octubre respectivamente, no llegando la media anual a $\operatorname{los} 300 \mathrm{~mm}$., mientras que invierno y verano son estaciones más secas y estables.

\section{Hidrografǐa}

El río Segura es el eje natural del territorio y su recorrido total es de $348 \mathrm{~km}$.. Aunque nace en la Sierra de Segura (provincia de Jaén), es el tercer río más importante de la Comunidad Valenciana después del Xúquer y el Túria. El caudal medio a su paso por la ciudad de Orihuela es de 15 $\mathrm{m} .{ }^{3} / \mathrm{seg}$. y el de su desembocadura en Guardamar del Segura alrededor de $8 \mathrm{~m} .3 / \mathrm{seg}$. (LÓPEZ GÓMEZ, 1977). El río origina una gran red de canales de riego y de drenaje del suelo, denominados respectivamente acequias o azarbes, que abastece a toda la huerta de la comarca. A pesar de su importancia para la agricultura, la calidad química del agua es deficiente debido a la constante y elevada contaminación por los vertidos industriales, las aguas fecales y los residuos sólidos urbanos que se vierten en su cauce. Por otra parte, las lagunas salobres de la Mata y Torrevieja están situadas al Sureste de la comarca y son las más extensas de España, existiendo en la segunda salinas cuya producción es la más grande de Europa. 

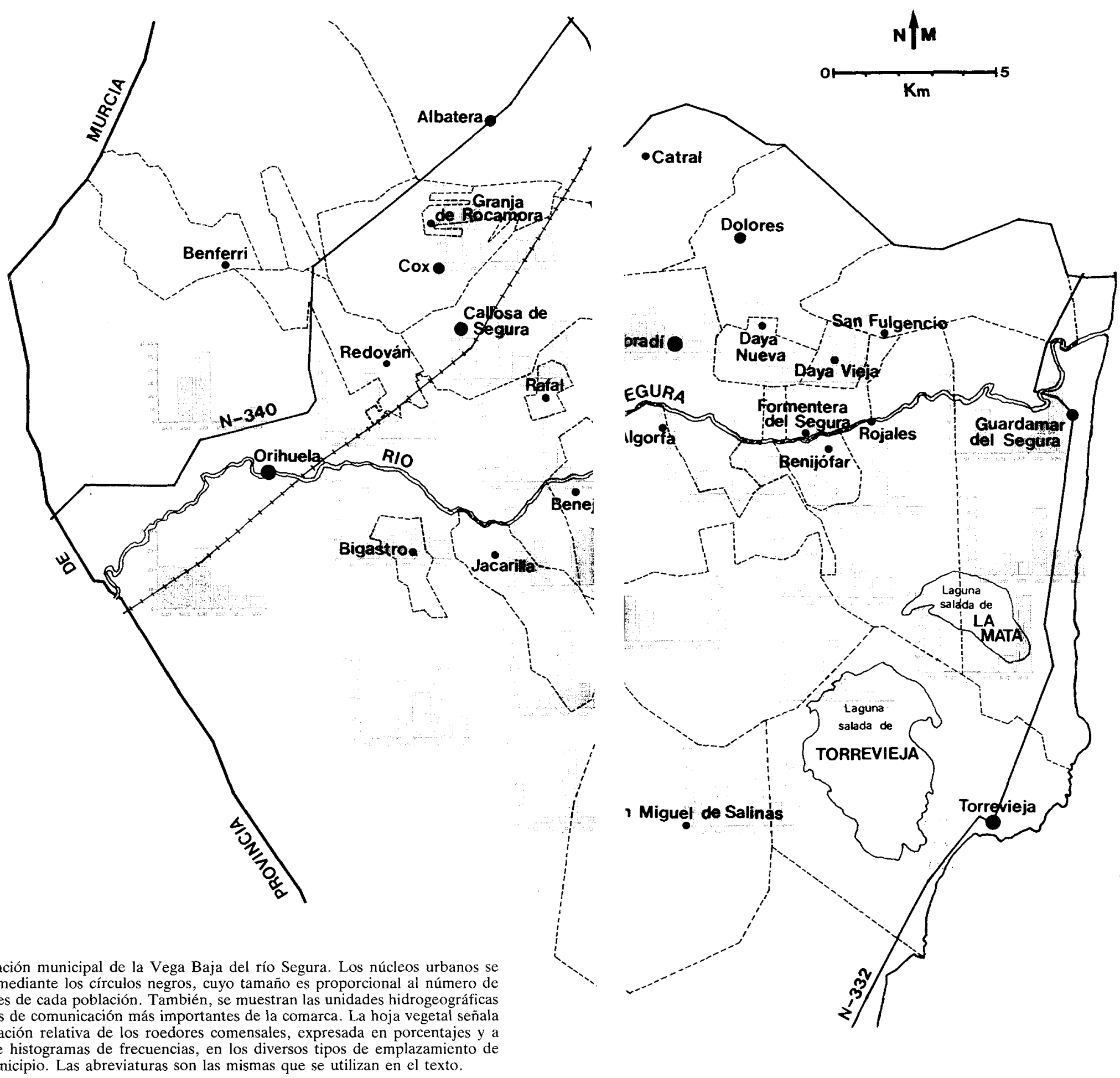

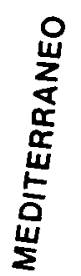

Figura 1.- Delimitación municipal de la Vega Baja del río Segura. Los núcleos urbanos se indican mediante los circulos negros, cuyo tamaño es proporcional al número de y las vías de coda población. Tambiên, se muestan las unidades hidrogeografical la infestación relativa de los roedores comensales, expresada en porcentajes y a cada municipio. Las abreviaturas son las mismas que se utilizan en el texto. 


\section{Relieve}

Geomorfológicamente, la zona está constituida por una gran depresión originada a partir del relleno de un golfo marino por los materiales sedimentarios del río Segura (RIBA, 1979), dando como resultado un delta interno no preeminente. El llano limita al Norte con las Sierras de Orihuela (605 m.) y Callosa $(561 \mathrm{~m}$.), que son de escasa extensión. Además, los materiales miocénicos meridionales del río Segura originan un peligroso frente de falla en su último tramo, produciendo históricamente una actividad sísmica importante (LÓPEZ GÓMEZ, 1977). El litoral presenta amplias playas y abundantes cordones de dunas.

\section{Vegetación}

La vegetación natural de la comarca se identifica con el espinar o matorral de palmito (Chamaerops humilis L.) y espino negro (Rhamnus lycioides L.) (Alianza Oleo-Ceratonion:'asociación Chamaeropo-Rhamnetum lycioides). Las áreas forestales se reducen a algunos bosquetes de pino carrasco (Pinus halepensis Mill.) que ocupan las Sierras de Orihuela y Callosa, donde la maquia o matorral de coscoja (Quercus coccifera L.) y lentisco (Pistacia lentiscus L.) (Alianza Oleo-Ceratonion: asociación Querco-Lestiscetum) se refugia en las umbrías y hondonadas. De importancia ornamental son los pinares y palmerales de algunas ciudades donde crecen $P$. halepensis, el pino rodeno (Pinus pinaster Aiton), la palmera datilera (Phoenix dactylifera L.), la palmera canaria (Phoenix canariensis hort. ex. Chaub.), el eucalipto (Eucaliptus globulus Labill.) y la pita (Agave americana L.). A orillas del río Segura y las acequias mayores son frecuentes los cañaverales (Arundo donax L.) y los carrizales (Phragmites communis Trin.), mientras que los matorrales de tamarisco (Tamarix gallica L) y adelfa (Nerium oleander L.) se distribuyen por ramblas y barrancos. La vegetación halófila en los alrededores de las lagunas de la Mata y Torrevieja corresponde a las praderas salinas (Clase Salicornietea). En las costas abunda la vegetación climácica de playas y dunas litorales (Clase Ammophiletea).

\section{Cultivos}

Según datos del INSTITUTO GEOGRÁFICO Y MINERO DE ESPAÑA (1978), la superficie total cultivada de la comarca es de 23.041 ha. de las que 13.586 ha. corresponden a cultivos herbáceos, 8.295 ha. a cultivos leñosos y 1.160 ha. a barbechos. Entre el arbolado de regadío destacan los agrios como el naranjo (Citrus aurantium L.) y el limonero (Citrus limon [L.] Burm. fil.). De los frutales de hueso predominan el almendro (Prunus dulcis [Mill.] D.A. Webb) y el olivo (Olea europaea L.). La explotación del granado (Punica granatum L.) y la higuera (Ficus carica L.) se lleva a cabo en algunas parcelas de Albatera y Orihuela. También sobresale el cultivo del trigo (Triticum vulgare Vill.) y las habas (Vicia faba L.). En 
TABLA 1

\begin{tabular}{lrrrrr}
\hline MUNICIPIO & \multicolumn{2}{c}{ CULTIVOS } & FORESTAL & OTROS & Total \\
\hline & Secano & Regadío & & & \\
\hline Albatera & & & & & \\
Algorfa & 403 & 4.340 & 1.170 & 1.396 & 7.309 \\
Almoradín & 547 & 773 & 327 & 225 & 1.872 \\
Benejúzar & 470 & 4.676 & 347 & 254 & 5.747 \\
Benferri & 30 & 457 & 308 & 70 & 865 \\
Benijófar & 319 & 769 & 66 & 65 & 1.219 \\
Bigastro & 42 & 260 & 99 & 39 & 440 \\
Callosa de Segura & 6 & 282 & 51 & 62 & 401 \\
Catral & 336 & 1.831 & 193 & 109 & 2.469 \\
Cox & 0 & 1.904 & 0 & 80 & 1.984 \\
Daya Nueva & 0 & 970 & 287 & 399 & 1.656 \\
Daya Vieja & 0 & 672 & 0 & 16 & 688 \\
Dolores & 0 & 294 & 0 & 4 & 298 \\
Formentera del Segura & 0 & 1.750 & 0 & 59 & 1.809 \\
Granja de Rocamora & 0 & 394 & 0 & 39 & 433 \\
Guardamar del Segura & 67 & 576 & 0 & 36 & 679 \\
Jacarilla & 878 & 1.810 & 1.155 & 908 & 4.751 \\
Orihuela & 175 & 877 & 77 & 107 & 1.236 \\
Rafal & 12.923 & 16.900 & 10.065 & 5.731 & 45.619 \\
Redován & 0 & 134 & 0 & 21 & 155 \\
Rojales & 70 & 574 & 0 & 338 & 982 \\
San Fulgencio & 19 & 1.855 & 413 & 936 & 3.223 \\
Total & 46 & 1.499 & 184 & 241 & 1.970 \\
\hline & 16.331 & 43.597 & 14.742 & 11.135 & 85.805 \\
\hline
\end{tabular}

Tabla 1.- Distribución municipal de la tierra en el año 1980 para cada uno de los municipios de la Vega Baja del río Segura (CAJA DE AHORROS DE ALICANTE Y MURCIA, 1980). Las cifras expresan la superficie en hectáreas.

la Tabla 1 se muestra la distribución municipal de la tierra en el año 1980 para cada uno de los municipios de la comarca (CAJA DE AHORROS DE ALICANTE Y MURCIA, 1980).

\section{MÉTODOS}

\section{Muestreo del medio físico}

El método seguido para muestrear el medio físico ha consistido en la inspección detallada de emplazamientos con características urbanas (MU) y rurales (MR). En ambos casos, la selección ha sido parcialmente al azar, incluyéndose con frecuencia lugares infestados indicados por los labradores. Las observaciones consideradas han sido directas cuando se vio directamente a los animales e indirectas si se observaron señales de su actividad como 
madrigueras, nidos aéreos, comederos, frezaderos, sendas y las comunicaciones verbales sobre su presencia. Las observaciones indirectas se evaluaron en raras, frecuentes y abundantes. Como emplazamiento entendemos cualquier construcción humana habitada o no, campos de cultivo, zonas de vegetación natural y cursos de agua.

Los emplazamientos del medio urbano se clasificaron en dos grupos según su situación respecto al casco urbano de cada municipio:

- MU1: emplazamientos ubicados en el interior de la población o en su periferia inmediata como acequias, alcachofales, almacenes de abonos y de piensos, depuradoras de agua, fábricas de conservas, granjas avícolas, mataderos municipales, naranjales, río Segura y vaquerías; y,

- MU2: emplazamientos ubicados en el área de influencia humana de cada población, cuyo radio de acción se consideró de $0.5 \mathrm{~km}$. y $1 \mathrm{~km}$. respectivamente para pueblos de hasta 2.500 habitantes y más de 2.500 habitantes. Aquí se contemplan acequias, alcachofales, alfafales, algodonales, almacenes de abonos y de piensos, almendrales, azarbes, campos de lechugas, depuradoras de aguas, fábricas de conservas, granjas de cerdos y de conejos, limonares, maizales, mataderos municipales, melonares, naranjales, palme-

TABLA 2

\begin{tabular}{lrrrrrrr}
\hline & MU1 & MU2 & MR1 & MR2 & MR3 & MR4 & TOTAL \\
\hline Albatera & 1 & 5 & 0 & 2 & 9 & 1 & 18 \\
Algorfa & 3 & 3 & 0 & 0 & 3 & 1 & 10 \\
Almoradí & 3 & 10 & 0 & 1 & 2 & 2 & 18 \\
Benejúzar & 2 & 4 & 1 & 2 & 3 & 0 & 12 \\
Benferri & 0 & 3 & 1 & 0 & 7 & 2 & 13 \\
Benijofar & 0 & 6 & 1 & 1 & 1 & 0 & 9 \\
Bigastro & 1 & 4 & 1 & 1 & 1 & 1 & 9 \\
Callosa de Segura & 1 & 5 & 0 & 2 & 4 & 0 & 12 \\
Catral & 3 & 4 & 1 & 1 & 3 & 1 & 13 \\
Cox & 4 & 2 & 1 & 1 & 4 & 2 & 14 \\
Daya Nueva & 1 & 1 & 0 & 1 & 4 & 1 & 8 \\
Daya Vieja & 1 & 3 & 0 & 1 & 3 & 1 & 9 \\
Dolores & 2 & 4 & 0 & 4 & 3 & 1 & 14 \\
Formentera del Segura & 3 & 3 & 0 & 4 & 3 & 1 & 14 \\
Granja de Rocamora & 1 & 0 & 1 & 1 & 7 & 1 & 11 \\
Guardamar del Segura & 1 & 4 & 1 & 3 & 5 & 4 & 18 \\
Jacarilla & 2 & 1 & 1 & 1 & 7 & 2 & 14 \\
Orihuela & 3 & 4 & 2 & 2 & 13 & 3 & 27 \\
Rafal & 2 & 5 & 0 & 1 & 2 & 1 & 11 \\
Redován & 1 & 6 & 1 & 2 & 3 & 2 & 15 \\
Rojales & 3 & 0 & 0 & 1 & 6 & 2 & 12 \\
San Fulgencio & 0 & 3 & 0 & 2 & 4 & 1 & 10 \\
Total & 38 & 80 & 12 & 31 & 99 & 31 & 291 \\
\hline
\end{tabular}

Tabla 2.- Número de emplazamientos inspeccionados en la Vega Baja del río Segura, distrubuidos por términos municipales y grupos de clasificación del medio físico. 
rales, patatales, presas fluviales, ramblas, río Segura, vaquerías y vertederos de basura.

A su vez, los emplazamientos del medio rural se dividieron en cuatro grupos según un gradiente regresivo de antropización cuya estimación fue subjetiva:

- MR1: vertederos de basuras;

- MR2: construcciones humanas habitadas o no como depuradoras de agua, estaciones hidráulicas, fábricas de baterías de automóviles y de conservas, granjas de cerdos y avícolas, mataderos municipales y vaquerías;

- MR3: campos de cultivos como ajares, alcachofales, alfafales, algodonales, almendrales, habares, higuerales, limonares, maizales, nabares, naranjales, olivares, parrales, patatales, plantaciones de granados, tomatales y trigales; $y$,

- MR4: zonas de vegetación natural y cursos de agua como acequias y azarbes, carrizales, dunas, golas del río Segura, palmerales, pinares, ramblas y el río Segura.

En la Tabla 2 se indica el número de emplazamientos inspeccionados de cada tipo en los términos municipales de la comarca.

La cartografía empleada se ha basado en el sistema UTM (Universal Transverse Mercator) con cuadrículas de $10 \times 10 \mathrm{~km}$. Las hojas cartográficas empleadas corresponden a las localidades de Elx, Fortuna, Guardamar del Segura y Orihuela, donde queda englobada la comarca.

\section{Tratamiento matemático de los datos}

El tratamiento matemático de los datos se ha considerado para tratar dos aspectos importantes entre los objetivos propuestos de este trabajo. A saber:

1) Por un lado, se ha procedido a evaluar la infestación de los roedores en los grupos de clasificación del medio físico o emplazamientos MU1, MU2 y MR1-MR4 en todos los municipios. Para ello, se ha elaborado un índice de infestación relativa, aplicable a investigaciones posteriores sobre plagas de roedores, y que viene definido por la fórmula siguiente:

$$
\mathrm{I}_{\mathrm{r}} \Sigma=\frac{\mathrm{Od}+\mathrm{Oi}}{\mathrm{n}} \text {, donde }
$$

$\mathrm{n}=$ número de emplazamientos de un mismo tipo,

$\mathrm{Od}=$ número de observaciones directas de los roedores, y

$\mathrm{Oj}=$ número de observaciones indirectas de los roedores a las que se asignó con carácter representativo el valor de 1 si son raras, 3 sin son frecuentes y 6 si son abundantes.

La cifra resultante se ha expresado en porcentajes respecto a la suma total de los valores del índice en cada tipo de emplazamiento de un municipio determinado. Como consecuencia de la presencia de las ratas y los ratones, se han registrado una serie de daños que hemos observado y eva- 
luado cualitativamente en los emplazamientos más infestados de cada término municipal.

2) Por otro lado, se ha aplicado el test $G$ de independencia entre variables (SOKAL Y ROHLF, 1979), previa elaboración de las tablas de contingencia pertinentes, utilizando el microordenador ECLIPSE C/350 del Centro de Cálculo de la Universitat de València (Burjassot, Valencia). Las variables estudiadas son "tipos de medio físico" (MF), "distancias al río Segura" (DR) y "especies de rata" (SP). Con anterioridad, se ha llevado a cabo una trasformación de los datos consistente en sumar 0.5 a todas las frecuencias de las casillas de cada tabla para evitar la presencia de casillas con ceros. El análisis fue aplicado sobre las modalidades de cada variable, tomando las observaciones directas, tal como se detalla a continuación: 1) respecto a los tipos de medio físico se han considerado el medio físico urbano y rural, así como los grupos MU1 y MU2 y MR1-MR4; 2) en relación con las distancias al río se distinguieron, desde su cauce hasta las zonas más alejadas del mismo por ambas márgenes, tres franjas paralelas de $5 \mathrm{~km}$. (A: de 0 a $5 \mathrm{~km}, \mathrm{~B}$ : de 5 a $10 \mathrm{~km}$ y C: de 10 a $15 \mathrm{~km}$ ); y, 3) por último, las especies de rata consideradas son $R$. norvegicus, $R$. Rattus y la rata de agua (Arvicola sapidus Miller, 1908).

\section{Técnicas complementarias para la obtención de datos}

Como complemento al muestreo de campo, se llevó a cabo una encuesta sobre la presencia de los roedores comensales en los municipios de la comarca. Se identificó cada emplazamiento afectado, las ratas y ratones presentes, la distribución y los daños originados. Asimismo, se han efectuado siete trampeos de eliminación con cepos en algunos emplazamientos representativos que, aunque insuficientes si consideramos la extensión de la región, han confirmado los resultados relacionados con la determinación de las especies y la ocupación de habitats por las mismas.

\section{RESULTADOS}

\section{Relación de especies}

Las observaciones directas obtenidas mediante el muestreo del medio físico han permitido previamente la determinación de varias especies de roedores. La especie que parece ser más frecuente entre las ratas es $R$. norvegicus, seguida de $R$. rattus y $A$. sapidus. La primera se encuentra más ligada a las poblaciones humanas que las otras dos pero, como a excepción de Orihuela todos los pueblos tienen pocos habitantes y se hallan inmersos en un ámbito estrictamente rural, las tres se distribuyen por doquier. Una especie de menor tamaño y costumbres silvícolas es el lirón careto (Eliomys quercinus Linnaeus, 1766), que ha sido citado por VERICAD, ESCARRÉ y RODRÍGUEZ (1976) como integrante de la dieta de la lechuza común (Tyto alba Scopoli, 1769) en tres localidades de esta comarca. 
La clasificación de ratones de la especie $M$. musculus se ha realizado directamente con ejemplares capturados vivos o muertos, pues sus señales de actividad más evidentes como son los excrementos se pueden confundir con los del ratón moruno (Mus spretus Lataste, 1883) o el ratón de bosque (Apodemus sylvaticus Linnaeus, 1758). Así, la especie más común y ubiquista es $M$. Musculus, mientras que $M$. spretus también es habitual aunque manifiesta tendencias más silvestres. VERICAD, ESCARRÉ y RODRÍGUEZ (1976) mencionan a M. musculus y al topillo común (Microtus duodecimcostatus De Sélys-Longchamps, 1839) en tres municipios, y a $A$. sylvaticus en dos de ellos.

\section{Distribución geográfica}

La mayor parte de la información recogida sobre la distribución geográfica de los roedores en la comarca procede de las observaciones directas y de los trampeos efectuados en algunas localidades. En la Tabla 3 se muestra el número de emplazamientos con observaciones directas de $R$. norvegicus, $R$. rattus y $A$. sapidus en cada término municipal para los grupos de

TABLA 3

\begin{tabular}{lccccccc}
\hline & MU1 & MU2 & MR1 & MR2 & MR3 & MR4 & TOTAL \\
\hline Albatera & $1 / 0 / 0$ & $0 / 0 / 0$ & - & $0 / 0 / 0$ & $0 / 0 / 0$ & $0 / 0 / 0$ & $1 / 0 / 0$ \\
Algorfa & $1 / 0 / 0$ & $0 / 0 / 0$ & - & - & $0 / 0 / 0$ & $1 / 0 / 0$ & $2 / 0 / 0$ \\
Almoradí & $0 / 0 / 0$ & $2 / 0 / 0$ & - & $0 / 0 / 0$ & $0 / 0 / 0$ & $0 / 0 / 0$ & $2 / 0 / 0$ \\
Benejúzar & $0 / 0 / 0$ & $2 / 0 / 0$ & $1 / 0 / 0$ & $1 / 0 / 0$ & $0 / 0 / 0$ & - & $4 / 0 / 0$ \\
Benferri & $0 / 0 / 0$ & $1 / 0 / 0$ & $0 / 0 / 0$ & - & $0 / 0 / 0$ & $0 / 0 / 0$ & $1 / 0 / 0$ \\
Benijófar & - & $0 / 0 / 0$ & $1 / 0 / 0$ & $0 / 0 / 0$ & $0 / 0 / 0$ & - & $1 / 0 / 0$ \\
Bigastro & $0 / 0 / 0$ & $0 / 0 / 0$ & $1 / 0 / 0$ & $1 / 0 / 0$ & $0 / 0 / 0$ & $0 / 0 / 0$ & $2 / 0 / 0$ \\
Callosa de Segura & $0 / 0 / 0$ & $1 / 0 / 1$ & - & $2 / 0 / 0$ & $0 / 0 / 0$ & $1 / 1 / 0$ & $1 / 1 / 0$ \\
Catral & $0 / 0 / 0$ & $0 / 0 / 0$ & $0 / 0 / 0$ & $0 / 0 / 0$ & $0 / 0 / 0$ & $1 / 1 / 0$ & $1 / 1 / 0$ \\
Cox & $0 / 0 / 0$ & $0 / 1 / 0$ & $1 / 0 / 0$ & $0 / 0 / 0$ & $0 / 0 / 0$ & $2 / 0 / 0$ & $3 / 1 / 0$ \\
Daya Nueva & $0 / 0 / 0$ & $0 / 0 / 0$ & - & $0 / 0 / 0$ & $0 / 0 / 0$ & $1 / 0 / 0$ & $1 / 0 / 0$ \\
Daya Vieja & $0 / 0 / 0$ & $0 / 0 / 0$ & - & $0 / 0 / 1$ & $0 / 0 / 0$ & $1 / 0 / 1$ & $1 / 0 / 2$ \\
Dolores & $0 / 0 / 0$ & $0 / 0 / 1$ & - & $0 / 0 / 0$ & $0 / 0 / 0$ & $0 / 0 / 0$ & $0 / 0 / 1$ \\
Formentera del Segura & $0 / 0 / 0$ & $1 / 0 / 0$ & - & $0 / 0 / 0$ & $1 / 1 / 0$ & $0 / 0 / 0$ & $2 / 1 / 0$ \\
Granja de Rocamora & $1 / 0 / 0$ & - & $1 / 0 / 0$ & $0 / 0 / 0$ & $0 / 0 / 0$ & $0 / 0 / 0$ & $2 / 0 / 0$ \\
Guardamar del Segura & $0 / 0 / 0$ & $0 / 0 / 0$ & $0 / 0 / 0$ & $1 / 0 / 1$ & $0 / 0 / 0$ & $1 / 1 / 0$ & $2 / 1 / 1$ \\
Jacarilla & $0 / 0 / 0$ & $0 / 0 / 0$ & $1 / 0 / 0$ & $0 / 0 / 0$ & $0 / 0 / 0$ & $0 / 0 / 0$ & $1 / 0 / 0$ \\
Orihuela & $1 / 0 / 0$ & $0 / 0 / 0$ & $1 / 0 / 0$ & $0 / 0 / 0$ & $0 / 0 / 0$ & $0 / 0 / 0$ & $2 / 0 / 0$ \\
Rafal & $0 / 0 / 0$ & $1 / 0 / 0$ & - & $0 / 0 / 0$ & $1 / 0 / 0$ & $0 / 0 / 0$ & $2 / 0 / 0$ \\
Redován & $0 / 0 / 0$ & $2 / 0 / 0$ & $0 / 0 / 0$ & $0 / 0 / 0$ & $0 / 0 / 0$ & $0 / 0 / 0$ & $2 / 0 / 0$ \\
Rojales & $1 / 0 / 0$ & - & - & $0 / 0 / 0$ & $0 / 0 / 0$ & $0 / 1 / 0$ & $1 / 1 / 0$ \\
San Fulgencio & - & $0 / 0 / 0$ & - & $0 / 0 / 0$ & $1 / 0 / 0$ & $1 / 0 / 0$ & $2 / 0 / 0$ \\
Total & $5 / 0 / 0$ & $10 / 1 / 2$ & $7 / 0 / 0$ & $5 / 0 / 2$ & $3 / 1 / 0$ & $8 / 3 / 1$ & $38 / 5 / 5$ \\
\hline
\end{tabular}

Tabla 3.- Número de emplazamientos de cada municipio con observaciones directas de ratas ( $R$. norvegicus $R$. rattus/A. sapidus) en la Vega Baja del río Segura, distribuidos por tipos de clasificación del medio físico. 
clasificación dèl medio físico. Los municipios con mayor cantidad de emplazamientos con ratas son Benejúzar, Callosa de Segura, Cox y Guardamar del Segura, mientras que los de menos son Albatera, Benferri, Benijófar, Daya Nueva, Dolores y Jacarilla. Las especies más observadas fueron $R$. norvegicus en Benejúzar, Callosa de Segura y Cox, y $A$. sapidus en Daya Vieja. Aunque en un número bajo de emplazamientos pero representativos a nivel local, $R$. rattus se ha encontrado en las localidades de Catral, Cox, Formentera del Segura, Guardamar del Segura y Rojales. El trampeo con cepos ha confirmado la existencia de $R$. norvegicus en los municipios de Callosa de Segura, Guardamar del Segura y Jacarilla. Asimismo, todas las observaciones indirectas han sido utilizadas para mostrar la distribución geográfica de las ratas, aunque las más fiables a nivel específico son el hallazgo de nidos aéreos de $R$. rattus, fenómeno ya estudiado con anterioridad en otras ocasiones (FAUS y VERICAD, 1981; FAUS, 1990). De este modo, los términos donde se han hallado con frecuencia estos artefactos son Albatera, Almoradí, Catral, Cox, Granja de Rocamora, Daya Vieja, Guardamar del Segura y Rojales. Sin embargo, según el resto de las observaciones indirectas cuyos resultados se presentan en la Tabla 4, la dispersión de las tres especies de rata en los municipios de la comarca es genera-

TABLA 4

\begin{tabular}{lcccccccc}
\hline & \multicolumn{9}{c}{ Observaciones directas } & \multicolumn{5}{c}{ Observaciones indirectas } \\
& R.n. & R.r. & A.s. & Mus sp. & R.n. & R.r. & A.s. & Mus sp. \\
\hline Albatera & + & - & - & - & + & + & + & + \\
Algorfa & + & - & - & - & + & + & + & + \\
Almoradi & + & + & - & + & + & + & + & + \\
Benejúzar & + & - & - & - & + & + & + & + \\
Benferri & + & - & - & + & + & + & - & - \\
Benijófar & + & - & - & - & + & + & + & - \\
Bigastro & + & - & - & + & + & - & + & + \\
Callosa de Segura & + & - & + & - & + & + & + & + \\
Catral & + & + & - & + & + & + & + & - \\
Cox & + & + & - & + & + & + & + & + \\
Daya Nueva & + & - & - & + & + & + & + & + \\
Daya Vieja & + & - & + & - & + & + & + & - \\
Dolores & + & - & - & - & + & + & + & - \\
Formentera del Segura & + & + & - & + & + & + & + & + \\
Granja de Rocamora & + & - & - & - & + & + & + & - \\
Guardamar del Segura & + & - & + & - & + & + & + & + \\
Jacarilla & + & - & - & - & + & + & + & - \\
Orihuela & + & - & - & + & + & + & + & + \\
Rafal & + & - & - & - & + & + & + & + \\
Redován & + & - & - & - & + & + & + & + \\
Rojales & + & + & - & - & + & + & + & + \\
San Fulgencio & + & - & - & - & + & + & + & + \\
\hline
\end{tabular}

Tabla 4.- Presencia de ratas $R$. norvegicus (R.n.), R. rattus (R.r.), A. sapidus (A.s.) y ratones (Mus sp.), considerando tanto las observaciones directas como las indirectas, en los términos municipales de la Vega Baja del río Segura. 
lizada, exceptuando Bigastro y Benferri donde faltan respectivamente $R$. rattus y $A$. sapidus. El trabajo efectuado por VERICAD, ESCARRÉ y RODRÍGUEZ (1976) aporta las citas de R. rattus en Algorfa, Dolores y San Miguel de Salinas, así como A. sapidus en Dolores. En la Tabla 4 puede verse indicada la presencia de $M u s$ sp. en cada localidad, a partir de las observaciones directas e indirectas. A pesar de no haber aparecido en todos los términos, el hallazgo en muchos emplazamientos de excrementos no identificables a nivel específico hace sospechar una distribución geográfica más extensa de las dos especies de ratones. Por último, VERICAD, ESCARRÉ y RODRÍGUEZ (1976) sitúan a $M$. musculus y $M$. duodecimcostatus en Algorfa, Dolores y San Miguel de Salinas, así como a A. sylvaticus en Dolores y San Miguel de Salinas.

\section{Emplazamientos ocupados}

Según se desprende de la Tabla 3, donde se muestra el número de emplazamientos con observaciones directas de las tres especies de rata, los más representativos pertenecen a los grupos MU2 y MR4. En concreto, $R$. norvegicus se localiza sobre todo en emplazamientos tipo MU2, MR4 y MR1, $R$. rattus en MR4 y $A$. sapidus en MU2 y MR2. Estos emplazamientos corresponden a vertederos de basuras, acequias y azarbes, río Segura, y menos frecuentemente a depuradoras de agua, fábricas de embutidos, granjas de cerdos y palmerales en el caso de $R$. norvegicus. Además, los palmerales y los limonares son biotopos donde $R$. rattus vive de modo habitual, desarrollando costumbres arborícolas. En muchos biotopos como los palmerales se ha observado a ambas especies viviendo juntas: $R$. norvegicus ocupa la parte inferior donde excava gran número de madrigueras subterráneas junto a los árboles, mientras que $R$. rattus construye nidos aéreos en la cruz y ramas entrelazadas de las palmeras. Por otro lado, $A$. sapidus se halla restringida a los canales de riego.

Respecto al género Mus, considerando tanto las observaciones directas como indirectas, parecen existir preferencias por los emplazamientos del tipo MU1. Particularmente, se ha comprobado la presencia de M. musculus en vertederos de basuras, almacenes de pienso, granjas de aves, el río Segura, azarbes y dunas. En cambio, $M$. spretus sólo fue observado en azarbes y el río Segura.

Los resultados del análisis de independencia entre las variables "tipos de medio físico", "distancias al río Segura" y "especies de rata", mediante la aplicación del test $\underline{\mathrm{G}}$ a las observaciones directas de las ratas, se muestran en la Tabla 5. Se observa un rechazo de dicha hipótesis en los casos MFxDRxSP ( $\underline{\mathrm{P}}<0.005,12$ grados de libertad) y MFxDR $(\underline{\mathrm{P}}<0.05,2$ g.l. $)$, pero no en el caso de MFxSP $(\underline{\mathrm{P}}<0.05,2$ g.l. $)$ y DRxSP $(\underline{\mathrm{P}}<0.05,4$ g.l. $)$. En otras palabras, se puede deducir que hay una dependencia global entre las tres variables estudiadas y que, independientemente de las especies de rata existentes, las distancias al río condicionan el tipo de emplazamiento 


\begin{tabular}{lcl}
\hline Hipótesis analizada & G.L. & $\underline{G}$ \\
\hline Independencia MFxDR & 2 & $16.341(\underline{\mathrm{P}}<0.005)$ \\
Independencia MFxSP & 2 & $1.194(\mathrm{NS})$ \\
Independencia DRxSP & 4 & $6.719(\mathrm{NS})$ \\
Independencia MFxDRxSP & 4 & $12.435(\underline{\mathrm{P}}<0.05)$ \\
Independencia MFxDRxSP & 12 & $36.688(\underline{\mathrm{P}}<0.005)$ \\
& NS: no significativo. \\
\hline
\end{tabular}

Tabla 5.- Test G de independencia entre las variables "tipos de medio físico" (MF), "distancias al río Segura" (DR) y "especies de rata" (SP), considerando las observaciones directas de los roedores en la Vega Baja del río Segura.

TABLA 6

\begin{tabular}{lcr}
\hline Hipótesis analizada & G.L. & $\underline{G}$ \\
\hline Independencia MFxDR & 10 & $319.964(\underline{\mathrm{P}}<0.005)$ \\
Independencia MFxSP & 10 & $115.982(\underline{\mathrm{P}}<0.005)$ \\
Independencia DRxSP & 4 & $12.382(\underline{\mathrm{P}}<0.05)$ \\
Independencia MFxDRxSP & 20 & $55.838(\underline{\mathrm{P}}<0.005)$ \\
Independencia MFxDRxSP & 44 & $504.164(\underline{\mathrm{P}}<0.005)$ \\
\hline
\end{tabular}

Tabla 6.- Test $G$ de independencia entre las "tipo variables de emplazamiento del medio físico" (MF), "distancias al río Segura" (DR) y "especies de rata" (SP), considerando las observaciones directas de los roedores en la Vega Baja del río Segura.

en el medio físico. Sin tener en cuenta esas distancias, la presencia de una $\mathrm{u}$ otra especie de rata no depende del tipo de medio y, sin considerar este último parámetro, la aparición de una u otra especie tampoco va asociada a las distancias respecto al río.

Finalmente, siguiendo la metodología del párrafo anterior, se han analizado las mismas variables pero considerando para la primera de ellas los tipos de emplazamiento del medio físico MU1, MU2 y MR1-MR4, cuyos resultados se exponen en la Tabla 6. En líneas generales, sé observan diferencias altamente significativas $(\underline{\mathrm{P}}<0.005)$ en todos los casos a excepción de DRxSP que son moderadas $(\underline{\mathrm{P}}<0.05)$. Se confirman los resultados expuestos más arriba, es decir, una dependencia generalizada entre los tres parámetros estudiados. Además, se encuentran asociadas entre sí las especies de rata con el tipo de emplazamiento y las distancias al río Segura, dejando aparte respectivamente las distancias al río y el tipo de emplazamiento. 


\section{Daños ocasionados}

A pesar de no evaluarse cuantitativamente los daños ocasionados por los roedores, sí se ha comprobado su incidencia en gran parte de los emplazamientos visitados, sobre todo en aquéllos donde se efectuó mayor número de observaciones de ratas y ratones. Los porcentajes de infestación relativa de las especies en los diversos tipos de emplazamiento de cada municipio de la comarca se presentan en la hoja vegetal de la Figura 1. Además, en la Tabla 7 se muestra el nivel de infestación municipal que se obtiene al sumar los valores de las observaciones directas e indirectas de los roedores en todos los emplazamientos y dividir por el número total de éstos, los daños observados en los emplazamientos más infestados y las especies que los han ocasionado. En líneas generales, $R$. norvegicus es la rata que provoca más daños, sobre todo destruyendo los márgenes de acequias, azarbes y alrededores inmediatos de las compuertas de control del agua, así como las bases de los muros de las construcciones humanas, por la excavación de madrigueras. También, este roedor puede incidir en la contaminación de forrajes en almacenes de piensos y granjas de cerdos y aves e incluso de alimentos para consumo humano en mataderos municipales, fábricas de embutidos y de conservas, por su mordisqueo o por el contacto con su orina o sus heces. En cambio, $R$. rattus, debido a sus costumbres más silvestres y a su tendencia a nidificar sobre los árboles (FAUS y VERICAD, 1981; FAUS, 1990), sería respectivamente la responsable principal de los daños locales en las edificaciones rurales y de la defoliación parcial de muchos frutales y de la ingestión de sus frutos. Esta especie se ha observado en los tejados y partes altas de las granjas de cerdos, así como sus nidos aéreos sobre Phoenix sp., C. aurantium, Pinus sp., setos de cipreses (Cupressus sempervirens L.), C. limon y membrilleros (Cydonia oblonga Mill.). No obstante, como $R$. norvegicus y $R$. rattus coexisten en muchos emplazamientos, por ejemplo en palmerales y granjas de animales domésticos, ambas serían responsables de los daños originados. En menor cuantía, pues su presencia no parece ser tan patente, $A$. sapidus causaría destrozos principalmente en las orillas de las acequias y azarbes por su actividad excavadora de madrigueras subterráneas.

Los problemas de los ratones pertenecientes a la especie $M$. musculus se centran en la contaminación de alimentos y roturas de sacos y embalajes en emplazamientos humanos como almacenes de piensos, de abonos y granjas de aves. En cambio, $M$. spretus puede causar pequeños daños muy localizados en algunas plantaciones hortícolas. Otros emplazamientos donde Mus sp. motivaría molestias similares son las fábricas de embutidos y las granjas de cerdos, y habría que controlar su presencia en los vertederos municipales y el río Segura pues éstos podrían constituir focos de dispersión hacia emplazamientos vecinos. 
TABLA 7

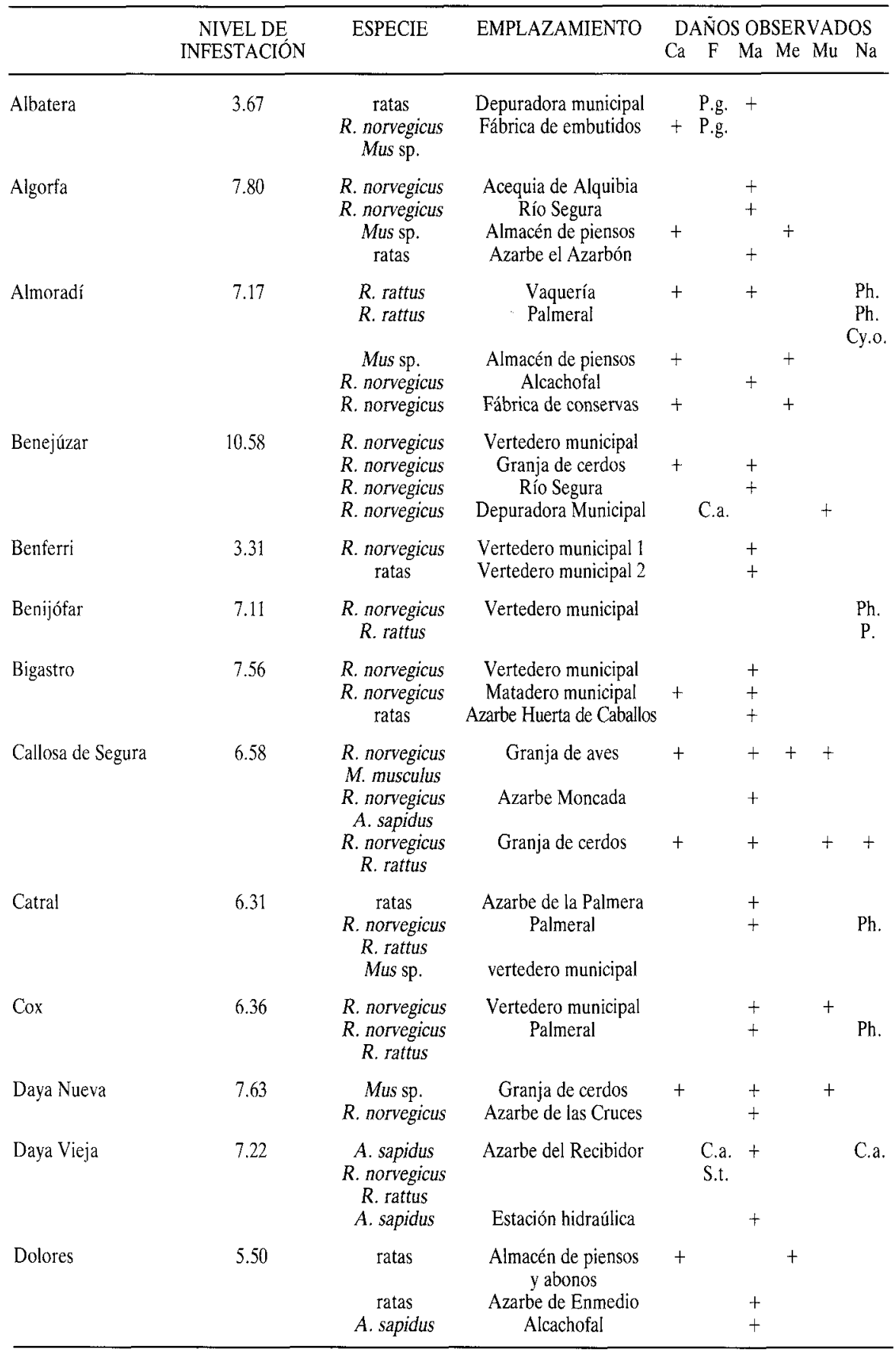


TABLA 7 (Continuación)

\begin{tabular}{|c|c|c|c|c|c|c|c|c|c|}
\hline & NIVEL DE & ESPECIE & EMPLAZAMIENTO & & AÑOS & $\mathrm{OB}$ & SER & VAD & \\
\hline & INFESTACIÓN & & & $\mathrm{Ca}$ & $\mathrm{F}$ & Ma & $\mathrm{Me}$ & $\mathrm{Mu}$ & $\mathrm{Na}$ \\
\hline Formentera del & & & & & & & & & \\
\hline Segura & 7.14 & $R$. norvegicus & Río Segura & & & + & & & \\
\hline & & ratas & Granja de cerdos & + & & + & & & \\
\hline & & R. rattus & Azarbe del Recibidor & & & + & & & C.a. \\
\hline & & R. norvegicus & Patatal & & & + & & & \\
\hline Granja de Rocamora & 4.73 & R. norvegicus & Vertedero municipal & & & + & & & \\
\hline & & R. rattus & Palmeral & & Ph. & + & & & Ph. \\
\hline & & $R$, norvegicus & Acequia de Arriba & & & + & & & \\
\hline Guardamar del & & & & & & & & & \\
\hline Segura & 5.00 & R. norvegicus & Fábrica de FEMSA & & & + & & & \\
\hline & & R. rattus & Limonar & & C.s. & + & & & C.s. \\
\hline & & & & & C.a. & ${ }^{\circ}$ & & & \\
\hline & & K. Tallus & Pinar & & & + & & & $P$. \\
\hline Jacarilla & 4.79 & $\begin{array}{l}R . \text { norvegicus } \\
\text { M. musculus }\end{array}$ & Vertedero municipal & & & + & & + & \\
\hline Orihuela & 4.85 & $R$. norvegicus & Vertedero municipal 1 & & & + & & + & \\
\hline & & R. norvegicus & Río Segura & & & + & & + & \\
\hline & & $\begin{array}{l}\text { M. musculus } \\
\text { ratas }\end{array}$ & $\begin{array}{l}\text { Almacén de piensos } \\
\text { Vertedero municipal } 2\end{array}$ & + & & $\begin{array}{l}+ \\
+\end{array}$ & + & + & \\
\hline Rafal & 7.00 & R. norvegicus & Vertedero municipal & & & + & & & \\
\hline & & R. norvegicus & Limonar & & C.1. & + & & & C.l. \\
\hline & & ratas & Azarbe de las Suertes & + & & & & & \\
\hline & & ratas & Fábrica de conservas & + & & + & + & & \\
\hline Redován & 3.93 & R. norvegicus & Fábrica de embutidos & + & & + & + & + & \\
\hline & & R. norvegicus & Depuradora municipal & & & + & & & \\
\hline Rojales & 7.17 & R. norvegicus & Río Segura & & & + & & & \\
\hline & & $\begin{array}{l}\text { M. musculus } \\
\text { M. spretus }\end{array}$ & & & & & & & \\
\hline & & $R$. rattus & Palmeral & & & + & & & Ph. \\
\hline & & ratas & Alcachofal & & & + & & & \\
\hline & & ratas & Acequia de la Alcudia & & & + & & & \\
\hline San Fulgencio & 6.40 & ratas & Limonar & & & + & & & \\
\hline & & R. norvegicus & Azarbeta la Roca & & & + & & & \\
\hline & & R. norvegicus & Tomatal & & & + & & & \\
\hline
\end{tabular}

Tabla 7.- Daños ocasionados por las ratas y los ratones en los emplazamientos más infestados en los términos municipales de la Vega Baja del río Segura. Se indica un nivel de infestación municipal resultante de sumar los valores de las observaciones directas e indirectas de todos los emplazamientos y dividir por el número total de éstos. Las abreviaturas de los daños se definen como sigue: Ca: contaminación de piensos y alimentos; F: frutos comidos (P.g. granadas; C.a. naranjas; S.t. patatas; Ph. dátiles; C.s. conos de ciprés y P. conos de pino); Ma: destrucción de márgenes de azarbes colindantes por excavación de madrigueras; Me: daños en sacos de piensos, abonos y embalajes de alimentos; Mu: destrucción de muros por excavación de madrigueras; y, Na: defoliación de árboles por construcción de nidos aéreos de $R$. rattus (Ph. Phoenix sp., Cy.o. Cydonia oblonga Mill., P. Pinus sp., C.a. C. aurantium, C.s. C. sempervirens y C.I. C. limon). 


\section{Encuesta}

La encuesta realizada sobre roedores comensales se ha dirigido al público en general, pero de manera especial a los agricultores de la comarca. Para ello, se diseñó un modelo original donde se cuestionaba en todos los grupos de clasificación del medio físico el tipo de roedor observado, su abundancia relativa, el lugar de observación (suelo o sitios elevados), las técnicas de control utilizadas y algunas consideraciones sobre este último factor. Los resultados correspondientes se presentan en la Tabla 8. Sobre el total de 100 cuestionarios recogidos, el número de respuestas por término municipal ha sido el siguiente: 7 en Almoradí, Dolores y Orihuela; 6 en Guardamar del Segura; 5 en Benejúzar, Cox, Formentera del Segura, Jacarilla y Rojales; 4 en Albatera, Algorfa, Benijófar, Bigastro, Callosa de Segura, Catral, Daya Nueva, Redován y San Fulgencio; y, 3 en Benferri, Daya Vieja, Granja de Rocamora y Rafal. Ante todo, cabe comentar que la denominación de las ratas y los ratones es confusa, pues las primeras son

TABLA 8

\begin{tabular}{|c|c|c|c|c|c|c|c|}
\hline & MU1 & MU2 & MR1 & MR2 & MR3 & MR4 & TOTAL \\
\hline \multicolumn{8}{|c|}{ OBSERVACIÓN DE } \\
\hline ratas & 9 & 16 & 6 & 26 & 12 & 5 & 74 \\
\hline ratones & 6 & 0 & 0 & 1 & 0 & 0 & 7 \\
\hline ratas + ratones & 6 & 5 & 0 & 5 & 2 & 1 & 19 \\
\hline SÍ muchos & 8 & 15 & 4 & 16 & 9 & 5 & 57 \\
\hline pocos & 13 & 6 & 2 & 16 & 5 & 1 & 43 \\
\hline NO (ninguno) & 0 & 0 & 0 & 0 & 0 & 0 & 0 \\
\hline \multicolumn{8}{|l|}{$\begin{array}{l}\text { LUGAR DE } \\
\text { ORSERYACIÓN }\end{array}$} \\
\hline suelo & 21 & 17 & 6 & 18 & 11 & 5 & 78 \\
\hline sitios elevados & 0 & 4 & 0 & 14 & 3 & 1 & 22 \\
\hline \multicolumn{8}{|l|}{ TÉCNICAS DE } \\
\hline manual & 0 & 0 & 0 & 0 & 1 & 1 & 2 \\
\hline cepos & 0 & 0 & 0 & 1 & 0 & 0 & 1 \\
\hline veneno & 20 & 19 & 5 & 29 & 13 & 5 & 91 \\
\hline otras & 1 & 2 & 1 & 2 & 0 & 0 & 6 \\
\hline \multicolumn{8}{|c|}{ ALTERNATIVAS DE } \\
\hline no & 3 & 6 & 1 & 2 & 1 & 1 & 14 \\
\hline ignora & 1 & 3 & 1 & 3 & 0 & 1 & 9 \\
\hline misma técnica & 7 & 5 & 2 & 15 & 1 & 1 & 31 \\
\hline técnica diferente & 10 & 7 & 2 & 12 & 12 & 3 & 46 \\
\hline TOTAL & 21 & 21 & 6 & 32 & 14 & 6 & 100 \\
\hline
\end{tabular}

Tabla 8.- Respuestas a la encuesta efectuada sobre el tipo de roedores observados, su abundancia relativa, el lugar de observación (suelo o sitios elevados), las técnicas de control utilizadas y alternativas a este factor en los emplazamientos inspeccionados en la Vega Baja del río Segura. 
conocidas mayoritariamente en la región por el nombre de ratones debido a su gran tamaño, mientras que los segundos se llaman ratas porque son más pequeños. En consecuencia, no es de extrañar que a nivel específico el desconocimiento taxonómico sea casi absoluto. Evidentemente, este hecho se ha tenido en cuenta a fin de evaluar correctamente las contestaciones de la encuesta. Según las respuestas recibidas, la opinión pública manifestó que las ratas (74 respuestas) suscitan una mayor problemática que los ratones (7), siendo la abundancia relativa de unos y otros poco variable, aunque en ningún caso se registró su ausencia absoluta. Se han comprobado más observaciones en el suelo (78) que en sitios elevados (22), correspondiendo respectivamente a las especies $R$. norvegicus, $A$. sapidus y Mus sp. y a $R$. rattus. En el suelo los emplazamientos más usuales donde se verificaron las observaciones han sido los márgenes de acequias y azarbes, los muros de granjas de animales, almacenes de piensos y de abonos, desagües, vertederos de basuras, paredes de casas de campo, cañaverales de $A$. donax, márgenes de caminos rurales y carreteras, orillas del río Segura, palmerales, muros de fábricas de embutidos y de conservas, depuradoras de agua y mataderos municipales. En sitios elevados cabe mencionar las vigas, techumbres y tuberías situadas en lo alto de granjas de animales y almacenes de piensos y de abonos, los nidos aéreos de $R$. rattus sobre $C$. aurantium, Phoenix sp., moreras (Morus nigra L.), setos de $C$. sempervirens, $C$. limon, $P$. dulcis y techumbres de fábricas de conservas. Los métodos de control más empleados para combatir a los roedores son mayoritariamente los venenos raticidas (91) y, en menor número, otros tipos de control como la ayuda de perros (Canis famialiaris Linnaeus, 1758) y gatos domésticos ( $\mathrm{Fe}$ lis catus Linnaeus, 1758) y la caza con armas de fuego (6), a golpes (2) y con cepos (1). Las alternativas sugeridas por muchas personas entrevistadas consideran oportuno un cambio de los métodos aplicados (46), aunque parte de ellas (31) prefieren seguir con las técnicas ya utilizadas.

\section{DISCUSIÓN}

La realización del presente trabajo en la Vega Baja del río Segura ha verificado la presencia generalizada de roedores como $R$. norvegicus, $R$. rattus, $A$. sapidus, $M$. musculus y $M$ spretus. Otras especies que también se han encontrado en la comarca son $E$. quercinus, $A$. sylvaticus y $M$. duodecimcostatus (VERICAD, ESCARRÉ y RODRÍGUEZ, 1976). La importancia faunística de los roedores comensales radica en que algunos como $R$. norvegicus y $R$. rattus constituyen posibles plagas para agricultura y salud pública por sus tendencias antropófilas (BROOKS, 1973), mientras que otros como $M$. musculus y $M$. spretus son presas básicas de la dieta de rapaces como $T$. alba (VERICAD, ESCARRÉ y RODRÍGUEZ, 1976; FAUS y GIL-DELGADO, 1987).

La distribución geográfica de las tres de rata abarca prácticamente todo el territorio, lo mismo que la de los ratones aunque sobre éstos falte infor- 
mación suficiente. Según las observaciones directas de los animales, $R$. norvegicus sólo está ausente de Dolores, $R$. rattus falta en Benejúzar, Benferri, Benijófar, Bigastro, Callosa de Segura, Daya Nueva, Jacarilla, Orihuela, Redován, Rafal y San Fulgencio, considerando además los nidos aéreos y las citas bibliográficas, y A. sapidus sólo se encuentra en Callosa de Segura, Daya Vieja, Dolores y Guardamar del Segura. Sin embargo, teniendo en cuenta también las observaciones indirectas, las tres especies parecen extenderse por todos los municipios exceptuando Bigastro y Benferri donde se ausentan respectivamente $R$. rattus y $A$. sapidus. Esta difusión tan amplia se debe a dos factores. Por un lado, a las necesidades ambientales de las especies: $R$. norvegicus habita preferentemente zonas bajas, con temperaturas moderadas y disposición constante de agua, adaptándose fácilmente al medio urbano y a la vida semiacuática en el medio rural; $R$. rattus se instala tanto en las poblaciones humanas como en el campo, debido a su carácter comensal y a su versatilidad ecológica; y, $A$. sapidus queda restringida a las masas fluviales de curso permanente (GOSÀLBEZ et al., 1985). $Y$, por otro, a que la extensa red de acequias y azarbes que dividen gran parte de la comarca, en otros tiempos una antigua zona pantanosa (LÓPEZ GÓMEZ, 1977), favorece su dispersión. En consecuencia, estos roedores son frecuentes en los cultivos hortícolas que se sitúan a ambas orillas del río, como sucede en arrozales, lagos y marismas de otras regiones costeras (VALVERDE, 1967; FAUS, 1985a, b; CONTE, 1986; VENTURA, 1988). En cuanto a los ratones, al igual que $R$. norvegicus, $M$. musculus está ligado estrictamente a los habitáculos humanos y a las cercanías del agua, contrariamente a $M$. spretus que tiende a ocupar biotopos más áridos (FAUS, 1985b; GOSÀLBEZ et al, 1985).

La ocupación de los diferentes tipos de emplazamiento por las ratas tiende a ser mayoritaria en los situados en un corto radio de acción de influencia humana alrededor de los núcleos urbanos y los de carácter más natural. Esto indica una gran diversidad de preferencias en la expansión de los roedores y su alto nivel de adaptación al medio físico del territorio estudiado, limitadas lógicamente por las aptitudes biológicas de cada especie. Por un lado, $R$. norvegicus abunda en los vertederos municipales de basuras ubicados frecuentemente cerca de las poblaciones (FAUS, 1985c; SANTINI et al., 1985) y $R$. rattus ocupa emplazamientos más silvestres como los palmerales (FAUS, 1990). Por otro, A. sapidus tiende a distribuirse en los emplazamientos periurbanos y en los cursos de agua alrededor de las construcciones habitadas o no del medio rural; esto no indicaría ninguna tendencia comensal de la especie sino que la red de acequias y azarbes llega incluso a lugares altamente antropizados, así como el origen y el carácter eminentemente agrícola de la comarca. Respecto a $M$. musculus parece ser que es un roedor que siempre va unido a la presencia del hombre, hallándose por doquier en los pueblos y casas de campo, mientras que $M$. spretus se inclina por emplazamientos más asilvestrados. Según el análisis matemático efectuado sobre las variables tipos de medio físico y de emplazamientos, distancias al río Segura y especies de rata, se concluye una dependencia 
general entre ellas. En primer lugar, las distancias al río condicionan lógicamente la presencia de cultivos de regadío o de secano, porque los primeros necesitan el agua de modo frecuente y abundante, al contrario que los segundos, lo que obliga respectivamente a su explotación cerca o lejos del cauce fluvial. En segundo lugar, la presencia de una u otra especie de rata no depende de que el medio físico sea urbano o rural, resultado que confirma una distribución uniforme de los roedores, y tampoco de las distancias al río. Sin embargo, si consideramos los diversos tipos de emplazamiento en un medio y otro, sí existe una asociación de éstos con las especies de rata y las distancias al río, lo que se entiende por las tendencias ecológicas de cada roedor.

Los roedores típicamente comensales como $R$. norvegicus, $R$. rattus y $M$. musculus producen daños principalmente en los lugares habitados por el hombre, bien sea en los pueblos o en el campo. En los primeros, tanto ratas como ratones causan pérdidas económicas al destruir alimentos y materiales almacenados en fábricas de embutidos y almacenes de piensos, con el consiguiente peligro sanitario; mientras, en las industrias $R$. rattus deteriora los tejados, $R$. norvegicus los desagües y ambas especies afectarían al sistema de cableado eléctrico. En el segundo, los daños son variados: las tres especies proliferan en fábricas de conservas y granjas de animales domésticos ocasionando todo tipo de desperfectos; $R$. norvegicus y $A$. sapidus destruyen los márgenes de muchos canales de riego y azarbetas alrededor de las parcelas de cultivo, al igual que señalan GOSÀLBEZ et al. (1984) en el Delta del Ebro (Tarragona); $R$. norvegicus y $R$. rattus merman las cosechas hortofrutícolas directamente al comer productos como naranjas, granadas, dátiles, limones, patatas y conos de ciprés y de pino; y, $R$. rattus contribuye a la defoliación de los frutales por la construcción de sus nidos aéreos (FAUS, 1990). Este último roedor también ocasiona graves pérdidas en las plantaciones de caña de azúcar del sur de la Península Ibérica (ZAMORANO, PALOMO y VARGAS, 1988) y daña los palmerales de cocos en las regiones tropicales (TAYLOR, 1972; JACKSON, 1977; WILLIAMS, 1985). Aunque A. sapidus se halla distribuida por la huerta, no se puede considerar estrictamente comensal porque su alimento preferido son las plantas silvestres acuáticas (CONTE et al., 1985; VENTURA, 1988), lo mismo que $M$. spretus por su dieta esencialmente granívora. Otros roedores que tampoco viven directamente a expensas del hombre, pero que pueden causar daños locales son $E$. quercinus, $A$. sylvaticus y $M$. duodecimcostatus. Los dos primeros se extenderían con preferencia en los cultivos de secano donde incidirían en los frutos almacenados de las casas de campo y en áreas de repoblación forestal (ASHBY, 1959). Según observaciones inéditas del autor, $M$. duodecimcostatus causa plagas endémicas en plantaciones jóvenes de $C$. aurantium de localidades valencianas como Llíria y Castelló de la Ribera; este micrótido roe las raíces de los naranjos llegándoles a causar su muerte, hecho que puede repetirse en las zonas citrícolas alicantinas donde se distribuya la especie. 
El conocimiento específico de los roedores por los habitantes de la comarca es escaso y confuso. Sin embargo, existe una motivación evidente de la opinión pública por los daños causados tanto en los emplazamientos humanos de los pueblos como en los cultivos agrícolas, considerando a las ratas más perjudiciales que a los ratones. Las técnicas de control empleadas mayoritariamente son los venenos rodenticidas a base de anticoagulantes y casi la mitad de las personas encuestadas son partidarias de cambiar el método empleado habitualmente, siendo ésto quizás consecuencia de la ineficacia de los productos utilizados y de las campañas de desratización realizadas hasta la actualidad.

Finalmente, a fin de evitar la proliferación y el impacto ambiental de los roedores comensales tanto en el aspecto económico como sanitario, se aconsejan algunas medidas alternativas de carácter paliativo y resolutivo. A corto plazo, se recomienda el desbroze periódico de los palmerales, de los márgenes y la higienización del agua del río Segura, de las acequias y azarbes grandes, sobre todo a su paso por los pueblos, así como prohibir el vertido de residuos sólidos de procedencia doméstica y animales muertos a los cursos de agua. A medio plazo, se aconseja continuar con las medidas ya iniciadas y la erradicación de los vertederos municipales incontrolados. $\mathrm{Y}$, a largo plazo, se sugiere también seguir con las normas anteriores y la canalización de acequias y azarbes, tanto en el interior como en las cercanías de las poblaciones, y la realización de los programas de control de roedores más adecuados que contemplen la inclusión de técnicas químicas y biológicas. Para ello, según las medidas a adoptar, habría que tener presente la colaboración de técnicos especializados que elaborasen los informes previos correspondientes a cada caso.

\section{AGRADECIMIENTOS}

Quiero expresar mi sincero agradecimiento a aquellas personas y entidades que han hecho posible la realización del presente trabajo. A la Caja de Ahorros de Alicante y Murcia, a través del Patronato "Ángel García Rogel" de Orihuela, la subvención del mismo mediante una beca de investigación. A la Cámara Agraria Provincial de Alicante y a J. M. Lloréns, Ingeniero Agrónomo, las facilidades ofrecidas. Al Dr. J. R. Vericad, Investigador Científico del C.S.I.C., las sugerencias aportadas. Y, al Dr. J. R. Acuña, Profesor Numerario de la Facultad de C.C. Biológicas de la Universitat de València, la ayuda prestada en el tratamiento matemático de los datos. 


\section{BIBLIOGRAFÍA}

ARATA. A.A., 1977. Resumée de la Conférence cojointe FAO/OMS/OEPP sur les rongeurs importants en agriculture et pour la Santé publique. Bull. OEPP, 7 (2): 159-162.

ASHBY, K. R., 1959. Prevention of regeneration of woodland by field mice (Apodemus sylvaticus L.) and voles (Clethrionomys glareolus Schreber and Microtus agrestis L.). Q.J. For, 63 (3): 228-236.

BERRY, R.J., 1981. Town Mouse, Country mouse: adaptation and adaptability in Mus domesticus (M. musculus domesticus). Mammal. Rev., 11 (3): 91-136.

BROOKS, J. E., 1973. A review of commensal rodents and their control. CRC Crit. Rev. Environ. Control, 3 (4): 405-453.

BROOKS, J. E.; ROWE, F. P., 1979. Commensal rodent control. Unpublished document. WHO/VBC/79.726, $109 \mathrm{pp}$.

CAJA DE AHORROS DE ALICANTE Y MURCIA, 1980. Alicante 1980. Datos y series estadísticas. Ser. Pub. Caja de Ahorros de Alicante y Murcia, Alicante, 147 pp.

CONTE, L., 1986. Estudio biológico de la rata común, Rattus norvegicus Berkenhout 1769 , en el. Delta del Ebro (Tarragona). Tesis de licenciatura, Universidad de Barcelona, 243 pp.

— FISAS, A.; VENTURA, J.; DE SOSTOA, F.J., 1985. Estudio de la dieta alimentaria de Arvicola sapjdus (Rodentia, Arvicolidae) en el Delta del Ebro (Tarragona). VII Reunión Bienal R. Soc. Esp. Hist. Nat. (Barcelona, 16-20 Sept. 1985): 240.

FAUS, F. V., 1985a. Los mamíferos de la Dehesa del Saler, la Albufera de Valencia y sus arrozales circundantes: II. Catálogo de especies. VII Reunión Bienal R. Soc. Esp. Hist. Nat. (Barcelona, 16-20 Sept. 1985): 251.

- 1985b. Los mamíferos de la Dehesa del Saler, la Albufera de Valencia y sus arrozales circundantes: I. Distribución geográfica. VII Reunión Bienal R. Soc. Hist. Nat. (Barcelona, 16-20 Sept. 1985): 252.

- 1985c. La fauna de vertebrados terrestres en vertederos urbanos de la provincia de Valencia. I. Inventario de especies. VII Reunión Bienal R. Soc. Esp. Hist. Nat. (Barcelona, 16-20 Sept. 1985): 256.

- 1990. Vegetal supports for the aerial nesting of Black rat (Rattus rattus) in the East of Spain. Mammalia, 54 (1): 147-152.

- y VERICAD, J. R. 1981. Sobre nidos aéreos de rata negra, Rattus rattus (Linnaeus, 1758), en el naranjal saguntino (Valencia). Mediterránea 5: 67-96.

- y GIL-DELGADO, J. A., 1987. Contribución al conocimiento de la dieta de Tyto alba en el Levante ibérico. Actas VII Bienal R. Soc. Esp. Historia Natural, Pamplona: 157-166.

GOSÀlbEZ, J., 1979. La Fauna dels Paìsos Catalans, en Geografia física dels Països Catalans (Panareda y Nuet eds.), Ed. Ketrés, Barcelona, $226 \mathrm{pp}$.

- BARRIOS, G.; FABREGUES, C.; GÖTZENS, G.; RUÍZ, S.; TORRELL, A., 1984. La rata común, Rattus norvegicus, en el Delta del Ebro. Daños ocasionados y eficacia de la campaña de desratización. Agricultura, 628: 900-904.

— LÓPEZ FUSTER, M.J.; GÖTZENS, G.; SANS COMA, V., 1985. El poblament dels petits mamífers (Insectivora i Rodentia) a Catalunya. Requeriments ambientals i distribució geogràfica. Butll. Inst. Cat. Hist. Nat., 52 (Sec. Zool, 6): 209-230.

GRATZ, N.G., 1984. The global public health importance of rodents, in The organization and Practice of Vertebrate Pest Control. Ed. A. C. Dubock, ICI Plant Protection Division: $413-435$.

INSTITUTO GEOGRÁFICO Y MINERO DE ESPAÑA, 1978. Las cuencas media y baja del río Segura, En Plan Nacional de Investigación de Aguas Subterráneas. Instituto Geológico y Minero de España, Madrid.

JACKSON, W.B., 1977: Evaluation of rodent depredations to crops and stored products. EPPO Bull., 7 (2): 439-458.

JIRSIK, J., 1955. Die Hausratte, Rattus rattus (Lineé, 1758) wieder in de Tschechoslowaquei. Säugetierk. Mitt, 3: 21-29.

KAHMANN, H.; HAEDRICH, B., 1957. Eine Untersuchung an Ratus rattus Linnaeus, 1758 (Mam., Rod.) auf der Insel Korsika. Zool. Anz., 158: 233-257.

LÓPEZ GÓMEZ, A., 1977. Geografía de les terres valencianes. Ed. 3 i 4 (Papers bàsics), Valencia, $263 \mathrm{pp}$.

PANAREDA, J. M.; NUET, J., 1979. El clima i les aigües del Països Catalans, en Geografia física dels Països Catalans (Panareda \& Nuet eds.), Ed. ketrés, Barcelona, 226 pp. 
PELIKAN, J., 1981. Patterns of reproduction in the house mice, en Biology of the House Mouse (Ed. R. J. Berry). Symposia of the Zoological Society of London. Academic Press, London, 47: 205-229.

SANTINI, L.; CROVETTI, A.; MALFATTI, P.; CHESI, F., 1985. Contributi al controllo di roditori nocivi alle aree urbane e suburbane. I. Bonifica delle discariche di rifiuti solidi da Rattus norvegicus Berk. (Rodentia, Muridae). REDIA, 68: 523-570.

SCHWARZ, E.; SCHWARZ, H. K., 1943. The wild and commensal stocks of the house mouse, Mus musculus Linnaeus. J. Mammal., 24: 59-72.

SOKAL, R.R.; ROHLF, F.J., 1979. Estadística para biólogos. H. Blume Ediciones, Madrid, $832 \mathrm{pp}$.

TAYLOR, K.D., 1972. Rodent problems in tropical agriculture. PANS, 18 (1): 81-88.

TELLE, H. J., 1966; Beitrag zur Kenntnis der Verhaltensweise von Ratten, vergleichend dargestelt bei, Rattus norvegicus und Rattus rattus, Z. Ang. Zool., 53: 129-196.

VALVERDE, J. A., 1967. Estructura de una comunidad de vertebrados terrestres. Mon. Est. Biol. Doñana, 1: $218 \mathrm{pp}$.

VENABLES, L. S. V.; LESLIE, P. H., 1942. The rat and mouse populations of corn ricks. J. Anim. Ecol., 11: 44-68.

VENTURA, J., 1988. Contribución al conocimiento del género Arvicola Lacépède, 1799, en el nordeste de la Península Ibérica. Tesis Doctoral, Universidad de Barcelona (Memória inédita).

VERICAD, J.R.; ESCARRÉ, A.; RODRÍGUEZ, E, 1976. Datos sobre la dieta de Tyto alba y Bubo bubo en Alicante (SE de Iberia). Mediterránea, 1: 49-59.

WILLIAMS, J. M., 1985: Interrelationships and impact on agriculture of Rattus species in the tropical South Pacific. Acta Zool. Fennica, 173: 129-134.

WORLD HEALTH ORGANIZATION, 1974. Ecology and control of rodents of public health importance. Wld. Hith. Org. Technn. Rep. Ser., 553: 42 pp.

YALDEN, D. W., 1980. Urban small mammals. J. Zool. London, 191: 403-406.

ZAMORANO, E.; PALOMO, L.; VARGAS, J., 1988. La rata negra (Rattus rattus Linneo, 1758) como plaga de los cultivos ibéricos de caña de azúcar. Detección, estima y control de los daños ocasionados. Bol. San. Veg. Plagas, 14: 227-240. 